\title{
An observational study of antibiotic treatment in paediatric patients suffering from LRTI and Pneumonia in a tertiary care hospital
}

\author{
Subhradipta Bhattacharyya $^{1}$, Manjushree Mohanty ${ }^{2}$
}

\begin{abstract}
${ }^{1}$ Department of Pharmacology, Hi-tech Medical College and Hospital, Rourkela, Orissa, India ${ }^{2}$ Department of Pharmacology, Kalinga Institute of Medical Science, Bhubaneswar, Orissa, India
\end{abstract}

Received: 22 November 2016 Accepted: 26 December 2016

\section{*Correspondence to:}

Dr. Subhradipta Bhattacharyya, Email:

subhradiptabhattacharyya@yaho o.co.in

Copyright: (C) the author(s), publisher and licensee Medip Academy. This is an openaccess article distributed under the terms of the Creative Commons Attribution NonCommercial License, which permits unrestricted noncommercial use, distribution, and reproduction in any medium, provided the original work is properly cited.

\begin{abstract}
Background: The observational study was conducted to evaluate the utilization of antimicrobials and sensitivity-resistance pattern in paediatric patients suffering from LRTI and Pneumonia. A prospective, non-interventional, pharmaco-vigilant study.

Methods: 97 patients suffering from LRTI and Pneumonia admitted in paediatric ward, NICU and PICU from September 2012 to February 2014 were evaluated. The research protocol was approved by Institutional Ethical Committee, KIMS. Data were collected from case sheets of patients from the ward as well as Medical Record and Data section. Data on a continuous scale was expressed as a mean along with standard deviation. Categorical data was expressed as percentage. Comparative statistical analysis was done by using student's t-test in respect of data measured on a continuous scale. All differences with $\mathrm{P}$ value below 0.05 were labelled as statistically significant.

Results: Among the 97 RTI cases 62 patients had pneumonia and LRTI in 35 patients. Antibiotics were found to be used in combination. Two drugs combination like, macrolide + B-lactam (11.4\%), cephalosporin + aminoglycoside (25.7\%), amino-penicillin+ aminoglycoside (15.5\%) and piperacillin + aminoglycoside $(15 \%)$ were used in severe infection. Three drugs combination like Ceftriaxone \pm sul+Amikacin+anti-MRSA/carbapenem (5.1\%), Macrolide (azithromycin and Clarithromycin) +Cephalosporin+Aminoglycoside $(10.1 \%)$ were used in very severe infections. Significant resistance was observed against B-lactam and Macrolides.

Conclusions: Very severe infections with bacteremia or multiorgan failure were effectively treated with administration of Macrolide, Linezolid and Carbapenem with Ceftriaxone/ Piperacillin + Amikacin.
\end{abstract}

Keywords: Antibiotic use, Combination therapy, Pneumonia

\section{INTRODUCTION}

Broad spectrum antimicrobials and improved supportive treatments are of immense importance in present healthcare system to cure devastating diseases like septicemia, meningitis or pneumonia in paediatric patients. ${ }^{1}$

As resistance to commonly used and in general cheaper antibiotics has become more frequent, newer broadspectrum antibiotics or combination therapy often are required. The expensive newer drugs have harmful effects on protective microflora and may even be toxic or less effective. ${ }^{2}$ Development of new antibacterial agents with activity against multi-drug resistant bacteria is therefore considered as a critical public health need.

One of the effective measures to circumvent growing resistance is to use the most appropriate narrower spectrum agent instead of broad-spectrum treatment when information on anti-microbial susceptibilities is available.

The antibiotic resistance profiles of MDR bacteria like GNB vary by species, geographic location, regional antimicrobial use, and hospital site (like, ICU Vs wards). ${ }^{3}$ 
Therefore extensive up to date data regarding antibiotic prescribing, sensitivity and resistance pattern are of utmost importance.

Keeping these things in mind, the present thesis work was started to evaluate patients suffering from pneumonia and LRTI admitted in Paediatric ward, NICU (Neonatal Intensive Care Unit) and PICU (Paediatric Intensive Care Unit).

Relatively fewer studies were conducted in Indian subcontinent in these fields of research till date. The present work was conducted to study antibiotic use and sensitivity-resistance pattern of infecting microbes.

\section{METHODS}

From September 2012 to February 2014 patients admitted in paediatric ward, PICU (Paediatric intensive care unit) and NICU (Neonatal intensive care unit) with provisional diagnosis of respiratory tract infection were evaluated.

The present study is a non-interventional, uncontrolled, open chart, pharmaco-epidemiological and pharmacovigilant study. It includes total 97 patients admitted in paediatric ward, NICU and PICU from September 2012 to February 2014.

The research protocol was approved by Institutional Ethical Committee, KIMS. Data were collected from case sheets of patients from the ward as well as Medical Record and Data section of the Indoor patients receiving antibiotics along with supportive medications with provisional or confirmed systemic infection of LRTI or pneumonia.

The primary efficacy parameters were respiratory rate, temperature, diastolic pressure, cough and rales, hypoxia and chest pain, $\mathrm{SpO}_{2}, \mathrm{FiO}_{2}$, sputum and consolidation. Secondary efficacy parameters like radiological assessment (CXR, USG chest, CT chest) or hematology (PBS or blood culture etc.) investigations. ${ }^{4}$ Data were collected on each parameters at the beginning, mid of treatment and end of treatment to evaluate the improvement of the patients (like resolution of pneumonic consolidation in chest X-ray or normalisation of blood counts etc). ${ }^{5}$

Outdoor patients, patients with severe renal impairment or hepatic failure or patients who died were excluded from this study.

\section{RESULTS}

\section{Patient demographic profile}

The particulars of demographic profile of the admitted patients can be depicted in Table 1 .
Table 1: Patient demographic profile.

\begin{tabular}{|ll|}
\hline Total no of patients 97 with M:F ratio $4: 1$ \\
\hline Male & 78 \\
\hline Female & 19 \\
\hline Mean age & $6.2 \pm 4.8(\mathrm{sd})$ Days \\
\hline Mean body wt. & $18.9 \pm 13.8(\mathrm{sd}) \mathrm{kg}$. \\
\hline Mean days of hospitalization & $8.95 \pm 4.8(\mathrm{sd})$ Days \\
\hline
\end{tabular}

Among the 97 RTI cases 62 patients had pneumonia and LRTI in 35 patients.

The pneumonia patients can be classified into Moderate to Severe pneumonia $(15.3 \%)$ Severe pneumonia. $(35.8 \%)$ and Very Severe pneumonia $(12.5 \%)$.

50 patients out of 62 of pneumonia remained uncomplicated during the treatment and released after remission within short periods. 12 patients $(12.4 \%)$ had complications of pneumonia, including parapneumonic effusions, necrotizing pneumonia and pneumothorax along with bacteremia or sepsis (Among them 4 patients had PTB), respiratory failure, septic shock, and multiorgan dysfunction. ${ }^{1}$ (Figure 1) 3 patients received ionotropes with severe septic shock.

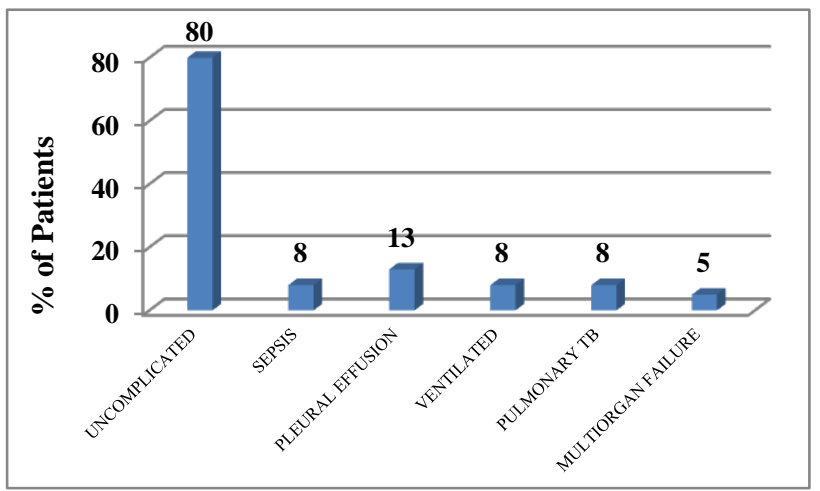

Figure 1: Complications of pneumonia and LRTI.

35 patients evaluated for LRTI, among them 5 patients were of very severe infection and received three antibiotics.

Pulmonary tuberculosis (4 patients), bronchial asthma, systemic infection like meningitis, AGE, UTI etc. were present in significant number of patients.

Therefore antibiotics were given intravenously at first according to severity (two antibiotic or three antibiotic combinations) and then switched to oral route when severity was decreased.

Antibiotics were prescribed in combination each and every time, all the patients received multiple antibiotics in combination, a single antibiotic was never found to be prescribed in this patients. ${ }^{6}$

Drug combinations can be categorized as below mention: 


\section{Two $\beta$ - lactam $\pm \beta-L I$ for moderate infection} (Pneumonia/ LRTI):

Two different drugs (like amoxicillin with cefuroxime) were used in $6(6.2 \%)$ patients of moderate pneumonia/ LRTI.

\section{Two drug combination for severe infection}

Two drug combinations were used in severe infections. These are:
a) Extended spectrum cephalosporin+ aminoglycoside $=25.7 \%$
b) Amino-penicillin + aminoglycoside $=15.5 \%$
c) Piperacillin + aminoglycoside $=15 \%$
d) Macrolide $+\beta$-lactam $=11.4 \%$ (Figure 2 ).

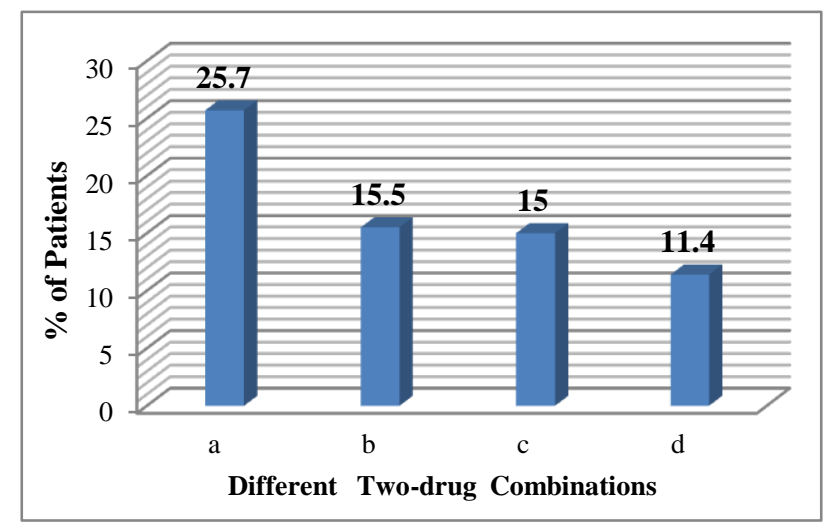

Figure 2: Two drug combination for severe infection.

\section{Three drug combination for very severe infection}

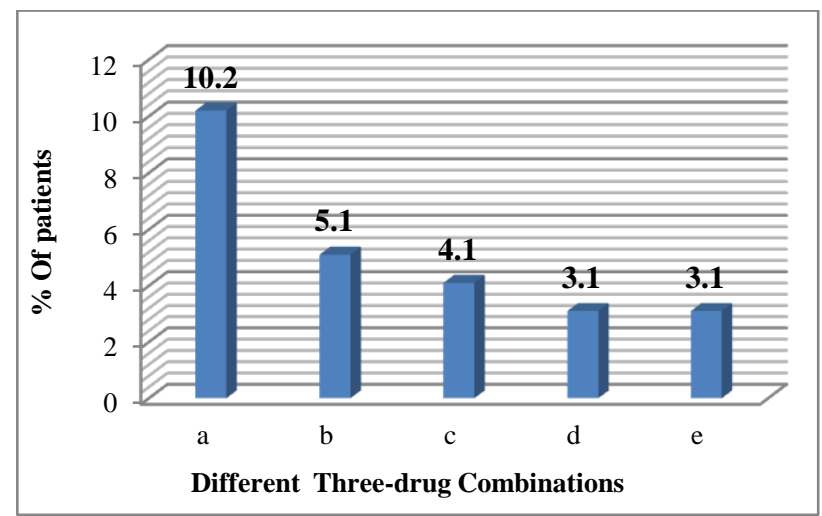

a=Macrolide $+\quad$ Cephalosporin $+\quad$ Aminoglycoside $\mathrm{b}=$ Ceftriaxone \pm sul+ Amikacin+anti-MRSA/ carbapenem. $\mathrm{c}=$ Amoxicillin + aminoglycoside + anti-MRSA/ carbapenem $\mathrm{d}=$ Piperaillin + aminoglycoside + anti MRSA/ carbapenem $\mathrm{e}=\beta$ Lactam+ anti-MRSA+ Metrogyl/ Ofloxacin

\section{Figure 3: Three drug combination for very} severe infection.

For very severe infection with/ without sepsis was treated with three or more than three drug combinations. These are: a) Macrolide (azithromycin/ clarithromycin)+ cephalosporin + aminoglycoside $=10 \quad(10.1 \%)$ patients

b) Ceftriaxone \pm sul+ amikacin+ anti-mrsa/ carbapenem $=5(5.1 \%)$ patients

c) Amoxicillin+ aminoglycoside+ anti-mrsa/ carbapenem $=4(4.1)$ patients

d) Piperaillin+ aminoglycoside+ anti $\mathrm{mrsa} /$ carbapenem $=3(3.1)$ patients

e) $\beta$-lactam+ anti-mrsa+ metrogyl/ ofloxacin $=3$ (3.1\%) patients (Figure 3).

\section{Duration of use of antibiotics}

Average use of different antibiotics is as- Linezolid (10 days), Piperacillin+tazobactum (9 days), Cephalosporin (8days), Aminoglycoside (7 days), Amoxi-clav (7 days) and Macrolide (3 days).

\section{Routes of administration}

- Most of the antibiotics were administered by I.V route ( $76 \%$ of total use).

- $\quad$ Antibiotics were prescribed by oral route (tab $10.5 \%$ or suspension/ syp $13.7 \%$ ) when infection was decreased by I.V. route initially ( $24 \%$ of total prescription).In Infants or $\leq 1-5$ years of age antibiotics were prescribed in form of Syrup. In children $\geq 7$ years old the drugs often were given in form of tablet.

\section{Use of individual antibiotics}

Cephalosporins were most frequently used (35.4\% of total antibiotic use) Cephalosporin was used on average for 8 days and I.V. twice or thrice daily.

- $\quad$ Ceftriaxone \pm sulbactam (59\%) was most frequently used followed by Cefuroxime (13\%), Cefoperazone \pm sulbactam $(11 \%)$, and Cefixime (9.7\%), Ceftazidime (3.3\%), Cefadroxil (2.2\%) and Cefotaxime (1.2\%) (Figure 4).

- Ceftriaxone was used in a dose of $40-50 \mathrm{mg} / \mathrm{kg} \mathrm{I.V}$ B.D for most of the patients though in a few patients of mild infection the drug was used in a dose of 20$25 \mathrm{mg} / \mathrm{kg}$ B.D.

- $\quad$ Cefuroxime was used in a dose of $10-13 \mathrm{mg} / \mathrm{kg} \mathrm{BD}$ in Tablet or Syrup form for severe pneumonia. In few cases lower doses $5 \mathrm{mg} / \mathrm{kg}$ BD in tablet form was used in RTI.

- $\quad$ Cefoperazone \pm sulbactum was used in dose of 45 $\mathrm{mg} / \mathrm{kg}$ BD I.V in most patients. Higher dose like 75 $\mathrm{mg} / \mathrm{kg}$ BD I.V was used in a few patients.

- Cefixime was used in a dose of $2.5-5.5 \mathrm{mg} / \mathrm{kg}$ BD orally.

- $\quad$ Ceftazidime \pm Tazobactum was used in a dose of 33$50 \mathrm{mg} / \mathrm{kg} \mathrm{BD} \mathrm{I.V.}$

- $\quad$ Cefotaxime was used in a dose of $50 \mathrm{mg} / \mathrm{kg} \mathrm{I.V} \mathrm{BD.}$

- Cefadroxil was used in syrup or tablet form. 


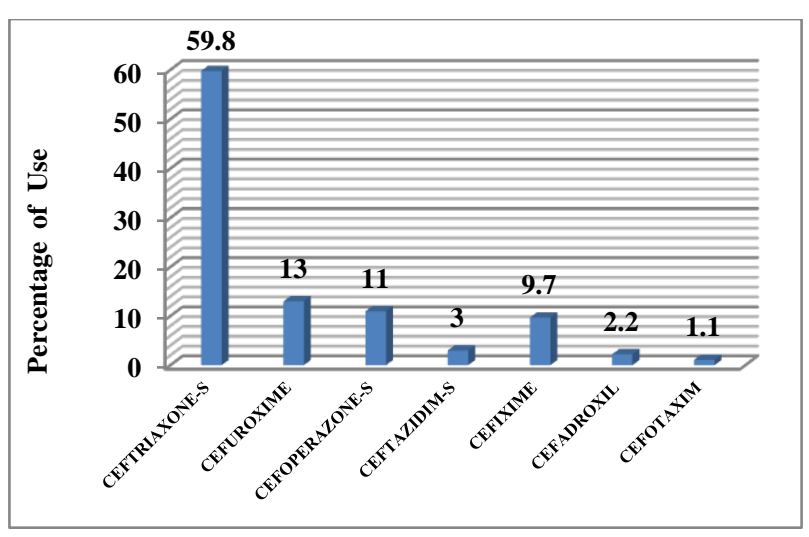

Figure 4: \% Use of different cephalosporins.

- Aminoglycoside (28.8\%) by i.v route was second most frequently used antibiotic. Aminoglycoside was used on average 7 days. The drug was given twice daily mostly, once daily in a few cases.

- Among the Aminoglycosides, Amikacin was used most of the time followed by Gentamicin and Netilmycin.

- $\quad$ Amikacin was used in a dose of 7-10 mg/kg I.V BD in most patients or $15 \mathrm{mg} / \mathrm{kg}$ I.V OD in few patients. For severe infections higher doses like $15-17 \mathrm{mg} / \mathrm{kg}$ I.V B.D was used.

- Gentamicin was used in the dose of $2-3 \mathrm{mg} / \mathrm{kg} \mathrm{I.V}$ BD or $4 \mathrm{mg} / \mathrm{kg}$ I.V OD.

- Netilmicin was used in a dose of $2.5-5 \mathrm{mg} / \mathrm{kg} \mathrm{I.V}$ $\mathrm{BD} / \mathrm{OD}$ in most of the patients.

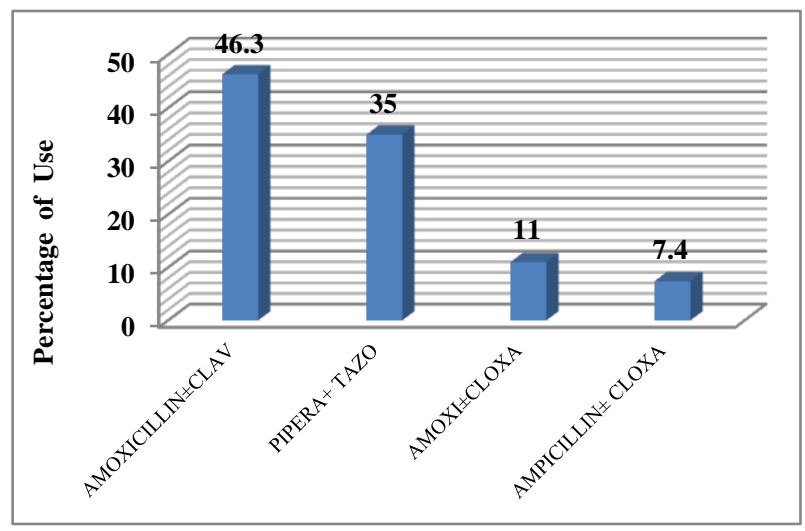

Figure 5: \% Use of different penicillins.

- In $21 \%$ patients Penicillin $\pm \beta$ LI were administered, firstly by i.v. route followed by oral route. In most cases penicillin were used in combination with Aminoglycosides.

- Amoxicillin+ clavulanic acid (use-9.6\% and failure rate-7.1\%) was used in dose of $35-50 \mathrm{mg} / \mathrm{kg} \mathrm{I.V}$ BD. Lower doses like $15-20 \mathrm{mg} / \mathrm{kg}$ I.V BD was given in a few patients.

- Piperacillin+ tazobactum (use-7.5\% and failure rate$14.8 \%$ ) was second most frequently used antibiotic, was used in dose of 70-110 mg/kg IV TDS.
- Amoxicillin \pm cloxacillin, Ampicillin \pm cloxacillins were used each in $1.7 \%$ patients.

- Amoxicillin+ Cloxacillin was used in dose of 50$100 \mathrm{mg} / \mathrm{kg}$ I.V TDS. Ampicillin was used in dose of 30-35 mg/kg I.V QDS. Ampicillin + Cloxacillin was used in dose of $45 \mathrm{mg} / \mathrm{kg}$ I.V TDS (Figure 5).

- Macrolides were used in $8 \%$ of RTI patients. Azithromycin was used in 17 patients and Clarithromycin was used in 4 patients in oral route.

- Azithromycin was used orally in Tablet or Syrup form in a dose of 10-20 mg/kg OD.

- Clarithromycin was used also both in Tablet and Syrup form in a dose of $10 \mathrm{mg} / \mathrm{kg} \mathrm{BD}$.

- $\quad$ ANTI-MRSA agents like Linezolid was used in $10(10.3 \%)$ patients. For severe infections the dose was $10 \mathrm{mg} / \mathrm{kg}$ I.V TDS. The dose was $5 \mathrm{mg} / \mathrm{kg}$ BD in less severe infection.

- $\quad$ Meropenem was used in $3.1 \%$ patients in a dose of 25-50 mg/kg I.V BD/TDS.

- Fluoroquinolones was not used in considerable percentage of cases.

Use of antifungal drugs: Used in $1 \%$ patients.

\section{DISCUSSION}

- After evaluation of resistance-sensitivity of isolated bacteria from different samples high resistance was observed against Penicillin $\pm \beta \mathrm{LI}$ and Ceftriaxone \pm $\beta L I$ (both gram +ve and -ve).

- In Gram negative isolates significant sensitivity was observed to Meropenem (72\%) and Aminoglycoside (65\%). 100\% sensitivity was observed to Vancomycin, Linezolid, Tigecycline and Quinpristin-dalfopristin in gram +ve isolates.

- This resistance-sensitivity pattern was reflected in mode of usage of the antibiotics. As an individual drug the Cephalosporins were stopped six times. Penicillin was changed in 7 patients. Aminoglycoside was changed five times whereas Piperacillin+tazobactam were stopped four times. Meropenem was stopped once.

- In few patients Piperacillin was replaced by Ceftriaxone.

- Two drug combinations have been used in severe infection. At first empirically two drug combination were started. Afterwards, according to c/s report in few cases or when adequate response was not seen, alteration in the regimen was done. Ceftriaxone \pm $\beta \mathrm{LI} /$ Cefoperazone $\pm \quad \beta \mathrm{LI}$ with Amikacin/ Gentamicin have been used in most of the patients (25.7\%). Piperacillin $\pm \beta L I$ and Amoxi/ ampicillin \pm $\beta \mathrm{LI}$ along with aminoglycoside have been used in $15 \%$ patinets. The antimicrobial spectrum of cefotaxime and ceftriaxone with or without aminoglycosides is excellent for the treatment of community-acquired pneumonia, i.e., that caused by 
some pneumococci (achievable serum concentrations exceed MICs for many or most penicillin-resistant isolates), $H$. influenzae, or $S$. aureus, Klebsiella etc. ${ }^{12}$

- Piperacillin \pm tazobactum extends the spectrum of ampicillin to include most strains of $P$. aeruginosa, Enterobacteriaceae, many Bacteroides spp., and E. faecalis.

- The antimicrobial spectrum of aminoglycoside mostly against aerobic gram negative bacilli, limited activity against gram positive and inactive against anaerobic infections. In combination with a cell wall-active agent, such as a penicillin or vancomycin, an aminoglycoside produces a synergistic bactericidal effect in vitro against enterococci, streptococci, and staphylococci. ${ }^{13}$

- Therefore, on addition of Amikacin/Gentamicin with various $\beta$-lactum severe pneumonia/LRTI were effectively treated.

- Three drug combinations were used in very severe pneumonia. In most of these cases either Linezolid or Azithromycin were added to the two-drug regimen.

- Macrolide (azithromycin/ Clarithromycin) were used in combination with Cephalosporin+ Aminoglycoside in 10 patients of very severe pneumonia.

- Cephalosporin+ Aminoglycoside+ anti-MRSA/ carbapenem was used in $5(5.1 \%)$ patients, Amoxicillin+ aminoglycoside+ anti-MRSA/ carbapenem was used in 4 cases and Piperacillin+ aminoglycoside+ anti MRSA/ carbapenem was used in 3 patients.

- Azithromycin/ Clarithromycin was used in combination with $\beta$ lactams from the beginning of treatment in few patients to cover atypical pathogens. ${ }^{7}$ In many patients Macrolides were added on at the time of discharge from hospital for suspected residual pneumonitis.

- $\quad$ Anti-MRSA like Linezolid was used as second line drug added to previous regimen to control infection in six patients. ${ }^{8,9}$ Among them 3 patients were having ATT and developed septic shock with longer duration of hospitalization.

- It was used in I.V route B.D in 6-7 patients at first and then in oral form when the patient was stabilized.

- In case of 2-3 patients were given linezolid empirically from the beginning who were suffering from very severe pneumonia along with other serious infection like meningitis and were in stage of septic shock at the time of admission. ${ }^{12}$

- $\quad$ Meropenem was used as second line drug in case of $3(3.1 \%)$ patients to treat multi-drug resistant gram negative bacilli like Pseudomonas, Klebsiella, Enterobactor or E. coli. However, two of them were suspected to have PTB. ${ }^{10}$

- Meropenem and linezolid were both used in one patient.
- In order to evaluate the comparative efficacy between two combination of three drugs (B-Lactam+ ßLI + Aminoglycoside + Macrolide or anti-MRSA) data of two groups of patients were compared. Mean duration of hospitalization (taken as efficacy parameter) of a group of patients $(n=6)$ with very severe pneumonia having Linezolid/ Carbapenem along with B-Lactam+ BLI+ Aminoglycoside as empirical therapy with sterile $\mathrm{B} / \mathrm{S}$ was found 10.6 days \pm 2.73 (sd).

- Second group of patients $(n=10)$ with very severe pneumonia having Macrolide along with B-Lactam+ BLI + Aminoglycoside as empirical therapy with sterile $\mathrm{B} / \mathrm{S}$ was found to have mean duration of hospitalization 13.6 days \pm 4.24 (sd).

- It was found that after compairing between these two values of mean duration of hospitalization, addition of macrolides to B-Lactam+ BLI + Aminoglycoside has been emerged as equally efficacious as linezolid/ Carbapenem ( $\mathrm{p}$ value $>0.05$, student's t-Test).

- Macrolides having wide gram negative/ gram positive/ atypicals coverage along with immuomodulatory action responsible for their synergistic action in combination have strengthened the treatment. ${ }^{7}$

\section{CONCLUSION}

In the research work it was tried to depict the prescribing pattern of antimicrobials in a systematic way. In spite of high resistance observed among isolated bacteria, 3rd generation Cephalosporin with aminoglycoside were the mainstay of treatment. Linezolid/ Meropenem and Macrolides augmented the recovery when added to empirical therapy in patients of very severe infection with sepsis.

\section{Funding: No funding sources \\ Conflict of interest: None declared}

Ethical approval: The study was approved by the Institutional Ethics Committee

\section{REFERENCES}

1. Theodore C, Sandora J Thomas. Community acquired pneumonia. Nelson Textbook of Pediatrics. Vol.2, Part XVII-XXXIII, $19^{\text {th }}$ Edn; 2008.

2. Harrison; Principle of Internal Medicine. $18^{\text {th }}$ Edition.

3. Bradley JS, Byington CL, Shah SS, Alverson B, Carter ER, Harrison C, et al. The Management of Community-Acquired Pneumonia in Infants and Children Older Than 3 Months of Age: Clinical Practice Guidelines by the Pediatric Infectious Diseases Society and the Infectious Diseases Society of America. Clinical Infectious Diseases. 2011;53:2576.

4. Shah SS, Dugan MH, Bell LM, et al. Blood cultures in the emergency department evaluation of childhood pneumonia. Pediatr Infect Dis J. 2011;30:475-79. 
5. Virkki R, Juven T, Mertsola J, et al. Radiographic follow-up of pneumonia in children. Pediatr Pulmonol. 2005;40:7-223.

6. Combination antibiotic therapy for communityacquired Pneumonia Jesus Caballero* and Jordi Rello Caballero and Rello Annals of Intensive Care. 2011;1:48. http://www.annalsofintensivecare.com/content/1/1/48

7. Goodman Gillman's Manual of Pharmacology and Therapeutics; $12^{\text {th }}$ edition. Chapter 44 .

8. Restrepo MI, Mortensen EM, Waterer GW, Wunderink RG, Coalson JJ, Anzueto A. Impact of macrolide therapy on mortality for patients with severe sepsis due to pneumonia. Eur Respir J. 2009;33:153-9.

9. Rubinstein E, Kollef MH, Nathwani D. Pneumonia Caused by Methicillin-Resistant Staphylococcus aureus Clinical Infectious Diseases. 2008;46:S37885.

10. Barton-Forbes M, Hawkes M, Moore D, et al. Guidelines for the prevention and management of community associated methicillin resistant Staphylococcus aureus (CA-MRSA): a perspective for Canadian health care practitioners. Can J Infect Dis Med Microbiol. 2006;17(C):1B-24B.

11. Sally A, Kate G. Clinical update on Linezolid in the treatment of gram positive infections. Infection and Drug Resistance. 2012;5:87-102

12. Tamma PD, Cosgrove SE, Maragakisb LL. Combination Therapy for Treatment of Infections with Gram-Negative Bacteria Clinical Microbiology Reviews. 2012;25,3:450-70.

13. Goodman and Gilman's the Pharmacological Basis of Therapeutics by Goodman, USA; 1985.

Cite this article as: Bhattacharyya S, Mohanty M. An observational study of antibiotic treatment in paediatric patients suffering from LRTI and Pneumonia in a tertiary care hospital. Int J Basic Clin Pharmacol 2017;6:323-8. 